\title{
Special Issue: Optimization and Stochastic Control in Finance, Journal of Optimization Theory and Applications
}

\author{
Bruno Bouchard $^{1} \cdot$ H. Mete Soner ${ }^{2} \cdot$ Nizar Touzi $^{3}$ \\ Published online: 11 October 2018 \\ ○) Springer Science+Business Media, LLC, part of Springer Nature 2018
}

The call for papers for the present special issue was launched with deadline 31 December 2016. After 20 months of reviews, we have collected a representative spectrum of the current state of optimization and control problems in finance. The field of financial mathematics is very much connected to the central topic of this journal as a large spectrum of the corresponding problems is formulated by means of optimization and optimal control theory. This is inherent to the nature of the topic as optimal decision making plays a central role in management, hedging, and risks control.

In the recent literature, there is an important interest in optimal decision making under agents interactions. This is again motivated by financial observations, namely by the important contagion and mimicking phenomena which played a crucial role during the last financial crisis. Important developments are achieved in stochastic differential games and the recently introduced topic of mean field games. The first two papers of this volume address the important issue of systemic risk by solving a Nash equilibrium problem with interacting agents optimizing their own decision in terms of interbank lending and borrowing, while taking the other's behavior as given. The paper by R. Carmona, J.-P. Fouque, S.M. Mousavi and L-H. Sun addresses the optimal lending and borrowing decision in a network of interacting banks. The authors formulate a finite-player linear-quadratic stochastic differential game with delay where the banks monitor the log-monetary reserves, the delay being due to the clearing of debt obligations. This allows to describe how the delay affects liquidity and systemic risk. The next paper by L.-H. Sun extends to the situation where the monetary reserve is modeled as a system of coupled Feller diffusions, and examines the effect of adding

Nizar Touzi

nizar.touzi@polytechnique.edu

1 CEREMADE, University Paris Dauphine, place du Maréchal de Lattre de Tassigny, 75775 Paris Cedex 16, France

2 ETH Zürich, Department of Mathematics, Rämistrasse 101, 8092 Zürich, Switzerland

3 Department of Applied Mathematics, CMAP, Ecole Polytechnique, Route de Saclay, 91124

Palaiseau Cedex, France 
liquidity on the financial stability of the network. The mean field game limit of large number of banks is also discussed.

The third paper in this volume considers a general equilibrium model with incomplete financial markets and numéraire assets. This is a more traditional model in mathematical economics which expresses market equilibria in terms of stochastic games. While the previous literature uses fixed points arguments, the authors provide existence by analyzing some corresponding variational inequalities.

The fourth paper by Possamaï and Mastrolia addresses the continuous time Principal-Agent problem in the context of model uncertainty for the agent. The Principal-Agent problem is a nonzero sum stochastic differential (Stackelberg) game which plays a central role in contract theory, using appropriate incentives to solve the moral hazard risk. The key argument is to use recent results from second-order backward stochastic differential equations in order to reduce the game problem into an individual control problem.

The next stream of papers addresses in various settings the problem of continuous time optimal dividend payment, which is central to the theory of corporate finance. A specific feature of this class of problems is that the dividend payment is a singular control. The paper by Y. Wang, L. Wang and K.L. Teo considers a class of stochastic differential games motivated by the optimal investment and dividend problem of an insurer under model uncertainty. The paper by E. Marciniak and Z. Palmowski considers the situation where the insurance premiums are surplus dependent and provide sufficient conditions for a barrier strategy of dividend payments to be optimal. The paper by J.-L. Perez, K. Yamazaki and X. Yu allow for capital injection in the optimal dividend problem and provide an explicit solution by using the fluctuation identities of the refracted-reflected Lévy process.

Another important topic introduced in the recent period is the problem of robust hedging which turns out to have close connections with optimal transport theory (with a martingale restriction) and the Skorohod embedding problem in probability theory. In particular, the paper by J. Claisse, G. Guo and P. Henry-Labordère revisits the Valois solution of the Skorohod embedding problem by tools from stochastic control theory. This allows to obtain the robust hedging of delta-hedged call options and opens the door for the multi-marginals extension of this solution. The paper by I. Guo and G. Loeper uses convex duality tools to derive bounds on volatility derivatives and provides numerical approximation methodology by least squares projections.

The paper by P. Guasoni and M. Weber adresses the problem of portfolio optimization under market impact. Portfolio optimization is at the origin of the connection between finance and optimization/control methods. In the present contribution, the authors examine the more realistic setting where prices are not linear in the executed volume, and obtain asymptotically optimal trading policies in explicit form. The paper by $\mathrm{H}$. Tie, H. Zhang, and Q. Zhang also addresses a portfolio optimization problem where the control consists in choosing among two assets which one to hold and which one to short. The optimal policy is characterized by threshold curves obtained by solving the associated Hamilton-Jacobi-Bellman equations.

The paper by C. Chen, Y. Chen, Y. Ouyang, and E. Pasiliao incorporates the importance sampling technique into the stochastic alternating direction method of multipliers, with application to a class of stochastic composite problems with linear 
equality constraint. This paper is somehow away from the strict application of control theory in finance and is, however, included in this volume in view of the importance of probabilistic numerical approximation technique in the field of financial mathematics.

Finally, the last two papers provide some methodological results for stochastic control problems with delay. E.Savku and G.W. Weber establish necessary and sufficient maximum principle for a stochastic control problem for Markov regime switching jump diffusion with delay model and illustrate the results in the context of an optimal consumption problem. The paper by N. Li, Y. Wang and Z. Wu considers the LinearQuadratic formulation of a stochastic control problem with delay. Under the delayed feature, the standard characterization by an appropriate Riccatti equation fails. The main result provides a new viewpoint based on the so-called relaxed compensator.

The various contributions to this volume give clear indications about the state of the literature on optimization and control in the community of financial mathematics. While previous literature focused mainly on individual optimization problems, the current literature is very much involved in the challenging problems of optimization among interacting agents. Of course, this was already addressed in the mathematical economics literature. An important feature of the recent literature is the use of a simplified game theoretic setting with continuous time decision making. Remarkably, the continuous time formulation of the stochastic game problems allows for more accessible solutions as it opens the door to the differential calculus in the time direction. The analysis also uses crucially recent results on path-dependent stochastic control problems. We expect that these tools will gain more simplification so as to cover more applications in the future. 